\title{
Male-Specific Long Noncoding RNA TTTY15 Inhibits Non-Small Cell Lung Cancer Proliferation and Metastasis via TBX4
}

\author{
I-Lu Lai ${ }^{1}$, Ya-Sian Chang ${ }^{1,2}$, Wen-Ling Chan ${ }^{1,3}$, Ya-Ting Lee ${ }^{1}$, Ju-Chen Yen ${ }^{1}$, Chin-An Yang ${ }^{4,5,6}$ \\ Shih-Ya Hung ${ }^{7}$ and Jan-Gowth Chang $1,2,3,5, *$ \\ 1 Epigenome Research Center, China Medical University Hospital, Taichung 404, Taiwan \\ 2 Department of Laboratory Medicine, China Medical University Hospital, Taichung 404, Taiwan \\ Department of Bioinformatics and Medical Engineering, Asia University, Taichung 413, Taiwan \\ Division of General Pediatrics, Children's Hospital of China Medical University, Taichung 404, Taiwan \\ College of Medicine, China Medical University, Taichung 404, Taiwan \\ Division of Laboratory Medicine, China Medical University Hsinchu Hospital, Hsinchu 302, Taiwan \\ 7 Graduate Institute of Acupuncture Science, China Medical University, Taichung 404, Taiwan \\ * Correspondence: d6781@mail.cmuh.org.tw; Tel.: +886-4-22052121 (ext. 2008); Fax: +886-4-22031029
}

Received: 19 June 2019; Accepted: 13 July 2019; Published: 15 July 2019

\begin{abstract}
Gender affects cancer susceptibility. Currently, there are only a few studies on $Y$ chromosome-linked long noncoding RNAs (lncRNAs), and the potential association between lncRNAs and cancers in males has not been fully elucidated. Here, we examined the expression of testis-specific transcript Y-linked 15 (TTTY15) in 37 males with non-small cell lung cancer (NSCLC), and performed circular chromosome conformation capture with next-generation sequencing to determine the genomic interaction regions of the TTTY15 gene. Our results showed that the expression levels of TTTY15 were lower in NSCLC tissues. Lower TTTY15 expression levels were associated with Tumor-Node-Metastasis (TNM) stage. A TTTY15 knockdown promoted malignant transformation of NSCLC cells. Based on the bioinformatics analysis of circular chromosome conformation capture data, we found that T-box transcription factor 4 (TBX4) may be a potential target gene of TTTY15. The RNA immunoprecipitation and chromatin immunoprecipitation results showed that TTTY15 may interact with DNA (cytosine-5)-methyltransferase 3A (DNMT3A), and the TTTY15 knockdown increased the binding of DNMT3A to the TBX4 promoter. We concluded that low TTTY15 expression correlates with worse prognosis among patients with NSCLC. TTTY15 promotes TBX4 expression via DNMT3A-mediated regulation. The identification of lncRNAs encoded by male-specific genes may help to identify potential targets for NSCLC therapy.
\end{abstract}

Keywords: TTTY15; lncRNA; non-small cell lung cancer; TBX4; methylation; DNMT

\section{Introduction}

Lung cancer is one of the most frequently diagnosed malignant tumors and remains the leading cause of cancer-related deaths worldwide [1,2]. According to the biological characteristics, there are two main types of lung cancer: non-small cell lung cancer (NSCLC; approximately 85\% of lung cancer cases) and small cell lung cancer (SCLC) [3,4]. Since diagnosis is usually made at advanced stages of the disease, the five year overall survival rate among NSCLC patients is under $20 \%[5,6]$. In addition, the overall survival rate is two-fold higher among women than among men. Nonetheless, there is no conclusive evidence to explain the difference in the survival rate between the genders. 
The protein-coding genes of the $\mathrm{X}$ and $\mathrm{Y}$ chromosomes have been relatively well characterized. Some studies have shown that the dysregulation of $X$ inactivation and the loss of the $Y$ chromosome are involved in several types of cancers. Human $\mathrm{Y}$ chromosome deletions and rearrangements are associated with various cancers, including prostate cancer [7,8], bladder cancer [9], male sex cord stromal tumors, lung cancer [10,11], and esophageal carcinoma [12], indicating the presence of oncogenes and tumor suppressor genes on this chromosome. Nevertheless, the chromosomal regions responsible for the production of noncoding RNAs are not well known.

Long noncoding RNAs (lncRNAs) are transcribed from thousands of loci in the mammalian genome and play a wide range of roles in gene regulation and other cellular processes, including mRNA splicing, RNA decay, translation, and chromatin remodeling. In addition to contributing to a normal physiology, lncRNA expression and function have been linked to the initiation and progression of cancer. Differences in cancer susceptibility are consistently associated with gender in cancer epidemiology [13]. Men have worse prognoses and a higher mortality rate than women do. Nevertheless, so far, the sex-related lncRNAs are poorly characterized and the potential link between these IncRNAs and cancer has not been studied.

The human Y chromosome can be divided into three regions: (a) male-specific regions of the $\mathrm{Y}$ chromosome (MSY), (b) pseudoautosomal regions (PAR1 and PAR2), and (c) heterochromatin in the Yq area [14]. The $Y$ chromosome contains more than 200 specific genes that are important for male sex determination, germ cell differentiation, and masculinization of various tissues [15]. On the other hand, the cellular function of many lncRNAs encoded in MSY has yet to be elucidated. Recently, a study revealed that an intergenic Y-linked lncRNA named lnc-KDM5D-4 (lysine demethylase 5D) is associated with fatty liver, atherosclerosis, and coronary artery disease in men [16]. There is currently only a limited amount of data available regarding the association between $\mathrm{Y}$ chromosome-linked lncRNAs and human phenotypes. Moreover, the regulatory mechanisms of these lncRNAs remain to be studied.

Testis-specific transcript Y-linked 15 (TTTY15, $5263 \mathrm{bp}$ ) is encoded in the chromosome region Yq11.21 of MSY. Some studies have shown that a fusion of the TTTY15 gene with USP9Y (TTTY15-USP9Y) predicts prostate biopsy results $[17,18]$ in multiple cases of hepatocellular carcinoma, lung cancer, and prostate cancer, suggesting that TTTY15-USP9Y fusion is a potential driver of carcinogenesis. Nevertheless, the function of TTTY15 itself in cells remains unknown. In this study, we investigated the mechanism of action of TTTY15 in the progression of NSCLC. We found that TTTY15 was significantly downregulated in NSCLC tissue samples compared to adjacent noncancerous tissues. Furthermore, decreased expression of TTTY15 was associated with poor prognosis among patients with NSCLC. Our data also indicate that a knockdown of TTTY15 promotes cell proliferation, cell cycle progression, migration, and invasion. In addition, investigation of the mechanism revealed that TTTY15 can target and affect T-box transcription factor 4 (TBX4) expression via DNA (cytosine-5)-methyltransferase 3A (DNMT3A)-mediated regulation. Our results show for the first time that TTTY15 participates in NSCLC progression.

\section{Results}

\subsection{TTTY15 Expression is Low in NSCLC}

To determine whether TTTY15 plays a role in NSCLC, we tested the expression of TTTY15 in 37 pairs of NSCLC tissue samples and their paired nontumor tissue samples. The data suggested that TTTY15 expression in tumor tissue was significantly lower than that in the matching nontumor tissue (mean delta cycle threshold (dCT) of tumors vs. normal tissue samples: 0.65 vs. $1.26, p<0.0001$; Figure 1A). To investigate the correlation between TTTY15 expression and the clinical features of the patients, NSCLC tissues were categorized into low TTTY15 expression group $(n=27)$ and high TTTY15 expression group $(n=10)$ on the basis of the mean value of TTTY15 expression levels. We found that the expression level of TTTY15 correlated with Tumor-Node-Metastasis (TNM) stage but not age or 
tumor size (Table 1). These results indicate that TTTY15 may participate in NSCLC tumorigenesis. Next, we investigated TTTY15 expression in NSCLC cell lines, including adenocarcinoma (A549, H441) and squamous cell lung cancer (H2170, H520) cell lines. The amelogenin of squamous cell lung cancer

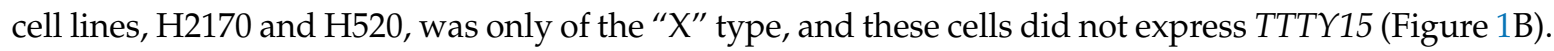
Thus, we chose A549 and H441 cells, which express amelogenin X and Y, for the following experiments.

A.
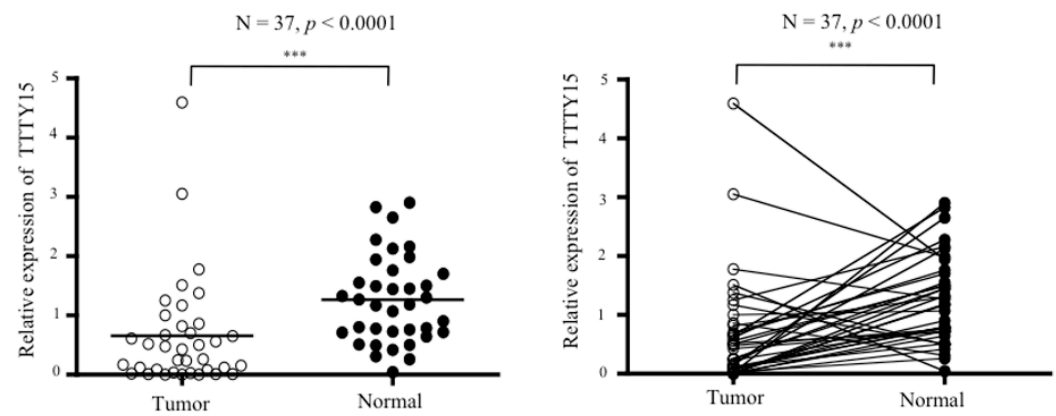

B.

C.
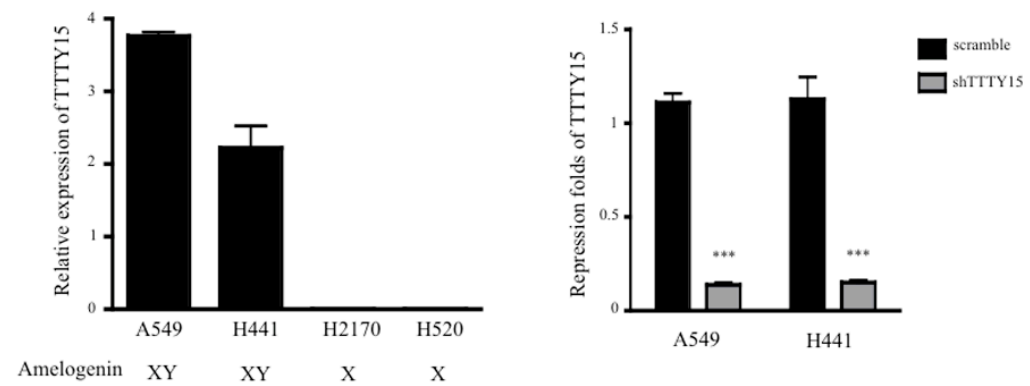

Figure 1. Relative expression and clinical significance of testis-specific transcript Y-linked 15 (TTTY15) in non-small cell lung cancer (NSCLC). (A) TTTY15 was found to be downregulated in human NSCLC compared to the adjacent normal tissue (left). The paired data for the TTTY15 expression in tumor and normal tissue is shown on the right ( $n=37$ for each group). (B) The relative expression levels of TTTY15 in NSCLC cell lines were validated by quantitative reverse-transcription PCR (qRT-PCR). (C) The efficiency of the TTTY15 knockdown in A549 and H441 cells was determined by qRT-PCR. Statistical analysis was based on Student's $t$-test $(n=3) ;{ }^{* * *} p<0.001$.

Table 1. TTTY15 expression and clinical characteristics of NSCLC patients $(n=37)$.

\begin{tabular}{|c|c|c|c|c|}
\hline \multirow[b]{2}{*}{ Parameter } & \multirow[b]{2}{*}{$n$} & \multicolumn{2}{|c|}{ Relative TTTY15 Expression } & \multirow{2}{*}{$p$ Value } \\
\hline & & $\begin{array}{c}\text { Low } \\
(n=27)\end{array}$ & $\begin{array}{c}\text { High } \\
(n=10)\end{array}$ & \\
\hline Age (years) & & & & 0.714 \\
\hline$\leq 65$ & 18 & 14 & 4 & \\
\hline$>65$ & 19 & 13 & 6 & \\
\hline Tumor size (maximum diameter) & & & & 0.453 \\
\hline$\leq 3 \mathrm{~cm}$ & 14 & 9 & 5 & \\
\hline$>3 \mathrm{~cm}$ & 23 & 18 & 5 & \\
\hline \multicolumn{5}{|l|}{ Lymph node metastasis } \\
\hline N1 & 25 & 21 & 4 & 0.0486 \\
\hline No & 12 & 6 & 6 & \\
\hline \multicolumn{5}{|l|}{ TNM (Tumor-Node-Metastasis) stage } \\
\hline I-II & 13 & 6 & 7 & 0.0167 \\
\hline III-IV & 24 & 21 & 3 & \\
\hline
\end{tabular}




\subsection{A Knockdown of TTTY15 Increased Lung Cancer Cells Proliferation and Cell Cycle Progression}

To determine the biological functions of TTTY15 in NSCLC cells, we first constructed stable knockdown cell lines shTTTY15-A549 and shTTTY15-H441 cells. The qRT-PCR data showed that TTTY15 was repressed by $\sim 90 \%$ in the two cell lines (Figure 1C). We then performed a 3-(4,5-dimethylthiazol-2-yl)-2,5-diphenyltetrazolium bromide (MTT) assay to test whether TTTY15 participated in cancer cell proliferation. The results showed that the knockdown of TTTY15 in NSCLC cell lines increased cell proliferation (Figure 2A). Next, to examine whether the effect of TTTY15 on cell proliferation reflects cell cycle progression, we performed a flow cytometry experiment to determine the cell cycle distribution. The results revealed that the knockdown of TTTY15 increased the prevalence of the G2-M transition among A549 and H441 cells (Figure 2B). These data indicated that TTTY15 may inhibit the proliferation of NSCLC cells.

A.
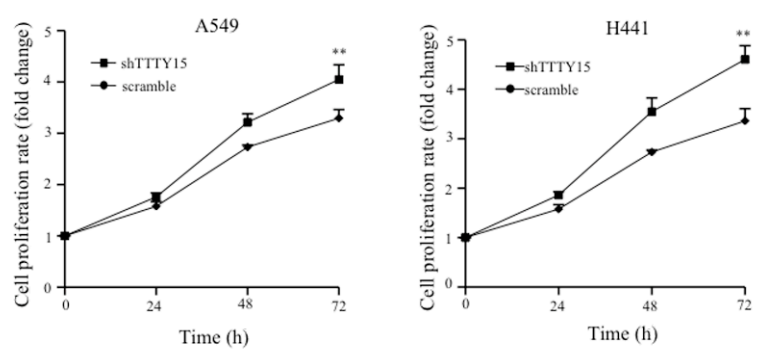

B.
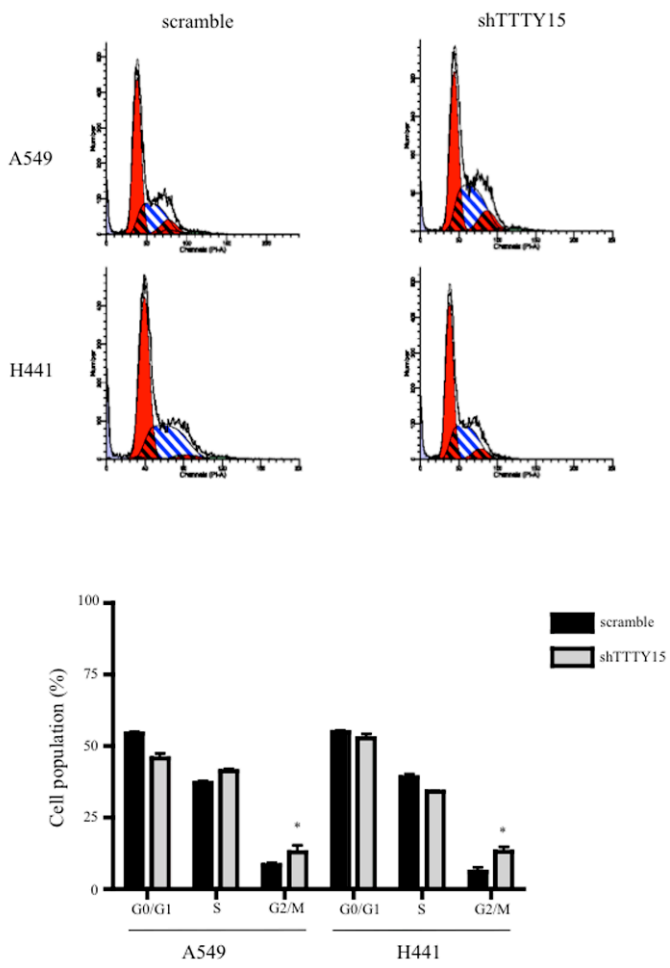

Figure 2. The TTTY15 knockdown increases cell proliferation and cell cycle progression. (A) The 3-(4,5-dimethylthiazol-2-yl)-2,5-diphenyltetrazolium bromide (MTT) assay shows that the TTTY15 knockdown increased the cell proliferation of A549 and H441 cells. (B) Representative images and results of quantification of the flow cytometry data. The cell cycle analyses revealed that TTTY15 influences A549 and H441 cell proliferation by regulating their cell cycle. The bar chart shows the percentage of cells in G0/G1 phases, in the S phase, and in G2/M phases. Data are presented as the mean \pm standard deviation (SD) of three independent experiments. All experiments were conducted in triplicate; ${ }^{*} p<0.05,{ }^{* *} p<0.01$. Statistical analysis was conducted by Student's $t$-test $(n=3)$. 


\subsection{The Knockdown of TTTY15 Increased NSCLC Cell Migration and Invasion}

To investigate the effects of TTTY15 on the migration of A549 and H441 cells, we performed a wound healing scratch assay. The data revealed that the proportion of the recovered region increased for the TTTY15 knockdown A549 and H441 cells as compared to the scramble cells (Figure 3A). Furthermore, we performed a Transwell invasion assay to evaluate the influence of TTTY15 on the invasiveness of NSCLC cells. The results indicated that the knockdown of TTTY15 significantly increased cell invasion as compared to the scramble cells (Figure 3B). These data meant that the knockdown of TTTY15 may promote cell migration and the invasive motility of NSCLC cells.

A.

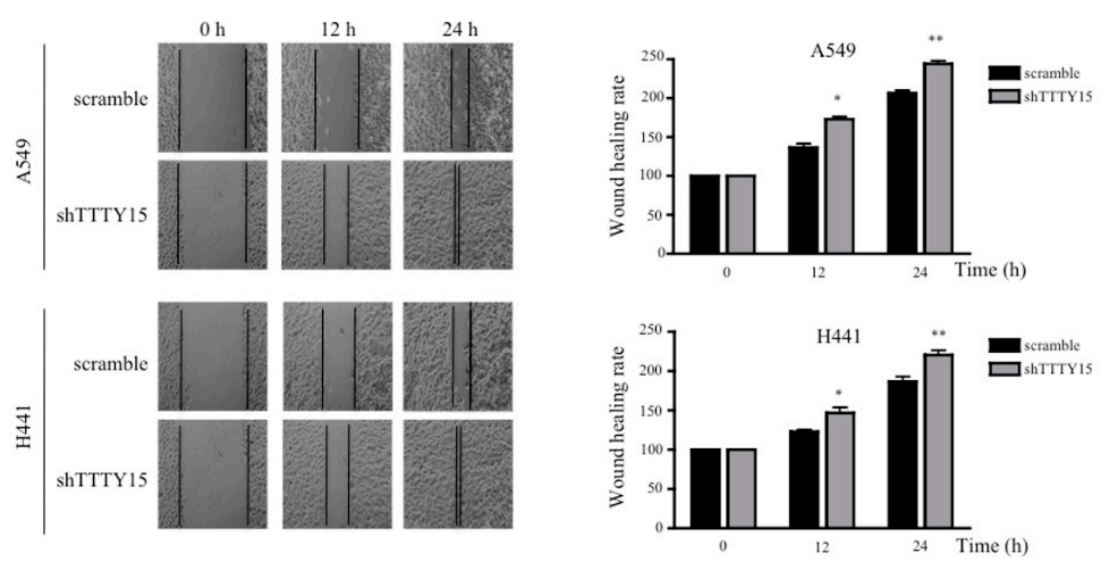

B.
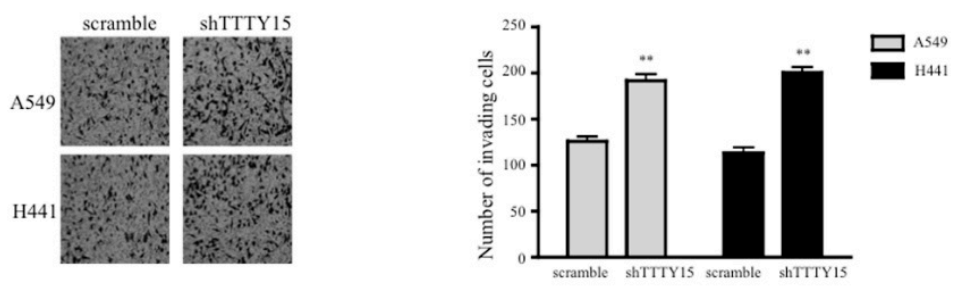

Figure 3. The TTTY15 knockdown increases cell migration and invasion. (A) Wound scratch assays were performed to analyze the migration efficiency of TTTY15 knockdown A549 and H441 cells compared to the scramble cells. The statistical analysis of the inhibition rates of A549 and H441 cells was performed at 12 and $24 \mathrm{~h}$, respectively. Magnification, $\times 200$. (B) The Transwell invasion assay was performed to determine the invasion ability of TTTY15 knockdown A549 and H441 cells compared to the scramble cells. Data are presented as the mean $\pm \mathrm{SD} ;{ }^{*} p<0.05,{ }^{* *} p<0.01$. Statistical analysis was conducted by Student's $t$-test $(n=3)$.

\subsection{TBX4 Is a Potential Target Gene of TTTY15 and Is Positively Regulated by TTTY15}

Recent studies showed that lncRNAs may modulate large-scale gene expression programs by interacting with chromatin at several thousand different locations across multiple chromosomes. To further explore TTTY15-driven regulation of cellular processes, we applied circular chromosome conformation capture (4C) coupled with next-generation sequencing to identify the TTTY15-interaction regions in A549 cells. The results indicated that TTTY15 might interact with KCNT2, WDR26, CCDC3, and LINC00674 (Table S1). After confirmation of the targeted genes using CviQ I and Sac I restriction enzyme sites, only CCDC3 and LINC00674 fitted the criteria. The trans-interactions between TTTY15 (located on the Y chromosome) with genes CCDC3 (chromosome 10) and LINC00674 (chromosome 17) are presented in Figure 4A. To determine the effects of TTTY 15 on these two target genes, we determined 
the expression levels of LINC00674 and CCDC3 in A549 and H441 cells. The data suggested that the knockdown of TTTY15 decreased the expression levels of LINC00674 but not CCDC3 (Figure 4B), indicating that TTTY15 may target and regulate LINC00674 expression. LINC00674, located in chromosomal region 17q23, is a long noncoding RNA gene, however, the function of LINC00674 is still unknown. Since lncRNA may regulate expression of genes on a large scale, we investigated whether TTTY15 could interact with this chromatin region and regulate the transcription of protein-coding genes located near LINC00674, including TBX4, KPNA2, and TIMP2 (Figure 4C). The results revealed that the knockdown of TTTY15 dramatically decreased the expression of TBX4 in A549 and H441 cells (Figure 4D). The spatial proximity of TTTY15 and LINC00674 chromatin segments was revealed through TTTY15 4C-sequencing and bioinformatic analysis. Moreover, TTTY15 was found to regulate the expression of TBX4 located near LINC00674.

A.

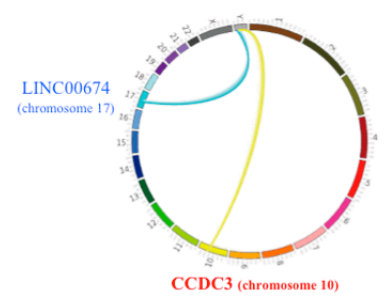

C.

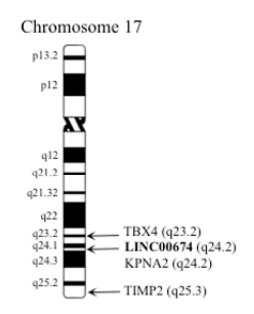

D.

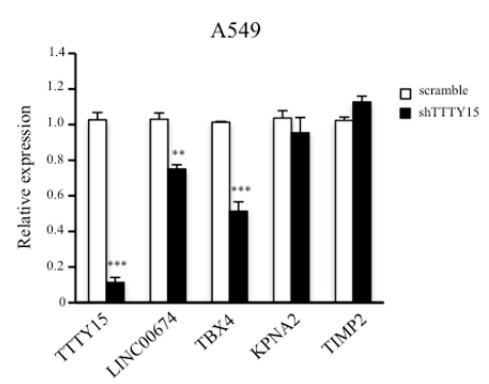

B.

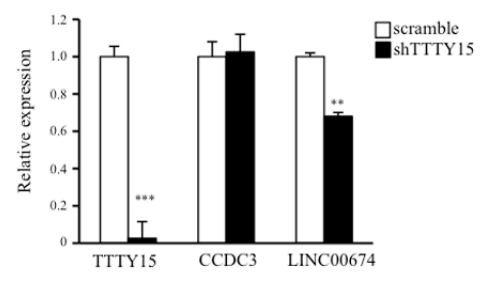

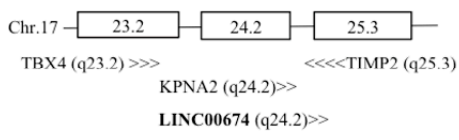

LINC00674 (q24.2)>>

Figure 4. T-box transcription factor 4 (TBX4) is a potential target gene of TTTY15 and its expression may be regulated by TTTY15. (A) The TTTY15 interaction map. (B) Comparison of the expression levels of CCCD3 and LINC00674 between TTTY15-knockdown cells and scramble cells. (C) The gene locus on chromosome 17 and a schema of TTTY15-targeted LINC00674 (bold) and the protein-coding genes near LINC00674. (D) Expression levels of protein-coding genes located near the LINC00674 gene in TTTY15 knockdown cells and scramble cells. Data are presented as the mean \pm SD; $p<0.05$, ${ }^{* *} p<0.01,{ }^{* * *} p<0.001$. Statistical analysis was conducted by Student's $t$-test $(n=3)$. 


\subsection{A Knockdown of TTTY15-Targeted TBX4 Increased Lung Cancer Cell Migration and Invasion}

Since the knockdown of TTTY15 inhibited TBX4 expression, we next elucidated the involvement of TBX4 in NSCLC. We determined the expression levels of TBX4 in NSCLC tissue samples and paired nontumor tissue samples. The data suggested that TBX4 was downregulated in tumor tissue samples (mean dCT of tumors vs. normal tissues: 0.01 vs. 0.06, $p<0.0001$; Figure $5 \mathrm{~A}$ ). We also investigated the correlation between TBX4 expression and the clinical features of the patients. NSCLC tissues were categorized into low TBX4 expression group $(n=28)$ and high TBX4 expression group $(n=9)$ on the basis of the mean value of TBX4 expression levels. We found that the expression level of TBX4 was related to TNM stage but not age or tumor size (Table S2). To further examine the role of TBX4 in NSCLC, a TBX4-specific small interfering RNA (siRNA called siTBX4) was designed and transfected into A549 and H441 cells. As depicted in Figure 5B, the cells transfected with siTBX4 showed significantly lower mRNA expression levels of TBX4 as compared to scramble control in both cell lines ( $p<0.01$; Figure 5B). In addition, the wound healing scratch assay and invasion assay suggested that the knockdown of TBX4 decreased the cell migration and invasion abilities (Figure 5C,D). We next tested whether TBX4 regulates the expression of matrix metallopeptidases (MMPs). The qRT-PCR results revealed that the TBX4 knockdown significantly increased the expression of MMP9 but not MMP2 (Figure 5E). These results indicated that the knockdown of TBX4 may promote NSCLC cell invasion and migration.

\subsection{TTTY15 Is Associated with DNMT3A and Decreases Its Binding to the TBX4 Promoter}

To investigate the potential mechanism by which TTTY15 regulates TBX4 expression in NSCLC, we analyzed the distribution of TTTY15 in cells. The cell fractional data indicated that TTTY15 was mainly localized in the nucleus (Figure 6A). Since other studies suggest that TBX4 is downregulated and hypermethylated in lung cancer-associated fibroblasts [19] and cancers, we speculated that TTTY15 may participate in the methylation status of the TBX4 promoter. We performed an RNA immunoprecipitation assay to determine whether there is a physical interaction between the DNA methyltransferases (DNMTs) and TTTY15. The results revealed that TTTY15 interacts with DNMT3A but not DNMT1 (Figure 6B). Therefore, we evaluated the expression level of DNMT3A in NSCLC. The results showed that the expression of DNMT3A in tumor tissue was higher than that in the nontumor tissue (mean dCT of tumors vs. normal tissues: 1.37 vs. $0.43, p<0.0001$; Figure $6 \mathrm{C}$ ). We also investigated the correlation between DNMT3A expression and the clinical features of the patients. We found that the expression of DNMT3A correlated TNM stage but not age or tumor size (Table S2). To test whether DNMT3A regulates the expression of TBX4 in NSCLC, DNMT3A was knocked down in A549 and H441 cells. The data revealed that the knockdown of DNMT3A increased the expression of TBX4 (Figure 6D). To elucidate the mechanisms of regulatory action of TTTY15, DNMT3A, and TBX4 in NSCLC, we performed Western blotting and chromatin immunoprecipitation (ChIP) on A549 and H441 cell lysates. The results indicated that the knockdown of TTTY15 did not affect DNMT3A protein expression (Figure 6E) but increased the binding ability of DNMT3A to the TBX4 promoter (Figure $6 \mathrm{~F}$ ). We then performed methylation-specific PCR to verify the CpG methylation status. The results revealed that knockdown of TTTY15 increased the methylated status in CpG islands (Figure 6G). Next, to determine whether the TTTY15-DNMT3A-TBX4 axis is involved in NSCLC, we knocked down DNMT3A and checked whether the cell migration and invasion abilities increased by shTTTY15 would be attenuated. The findings showed that the knockdown of DNMT3A indeed inhibited the cell migration and invasion in A549/shTTTY15 and H441/shTTTY15 cells (Figure S1). 
A.

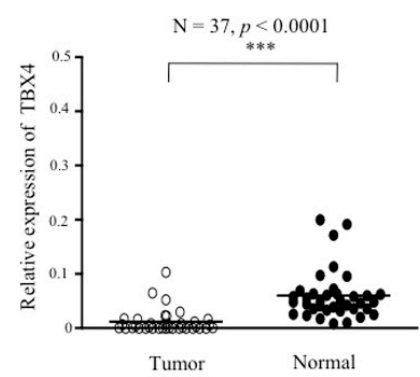

C.

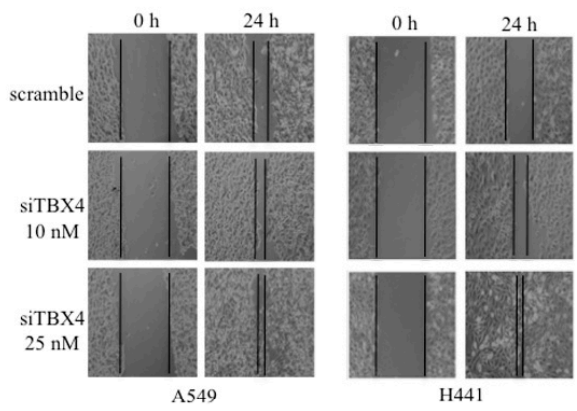

D.

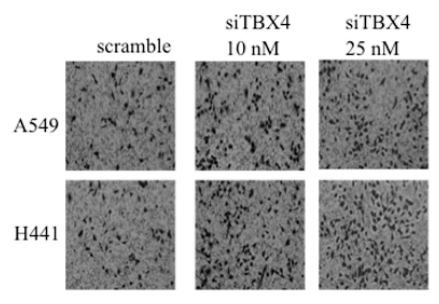

E.

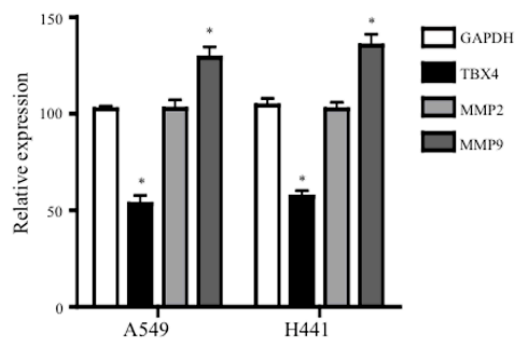

B.
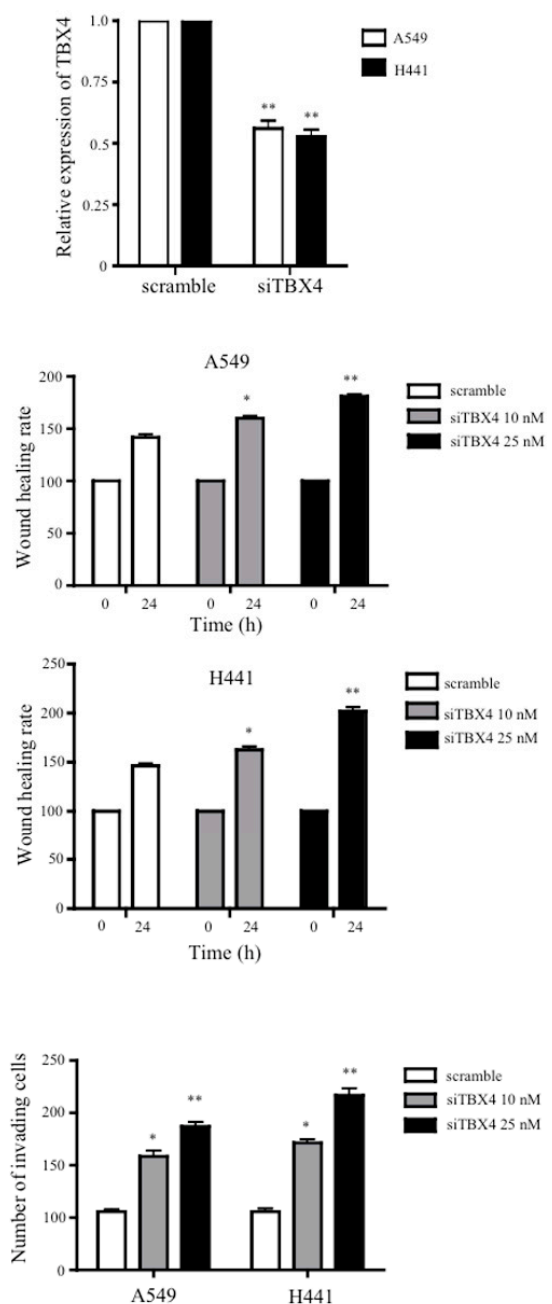

Figure 5. TBX4 was found to be downregulated in NSCLC, and the knockdown of TBX4 increased cell migration and invasion. (A) TBX4 is under-expressed in human NSCLC compared to the adjacent normal tissue ( $n=37$ for each group). (B) qRT-PCR was carried out to analyze the TBX4 knockdown efficiency of TBX4-specific small interfering RNA (siTBX4) in A549 and H441 cells. (C) Wound scratch assays were performed to analyze the migration abilities of TBX4 knockdown A549 and H441 cells compared to the scramble cells. The statistical analysis of the inhibition rates of A549 and H441 cells were performed at $18 \mathrm{~h}$. Magnification, $\times 200$. (D) Transwell invasion assays were performed to determine the invasion ability of TBX4 knockdown A549 and H441 cells compared to the scramble cells. (E) The knockdown of TBX4 increased the expression levels of matrix metallopeptidase (MMP) 9. Data are presented as the mean $\pm \mathrm{SD} ;{ }^{*} p<0.05,{ }^{* *} p<0.01$. Statistical analysis was conducted by Student's $t$-test $(n=3)$. 
A.

C.
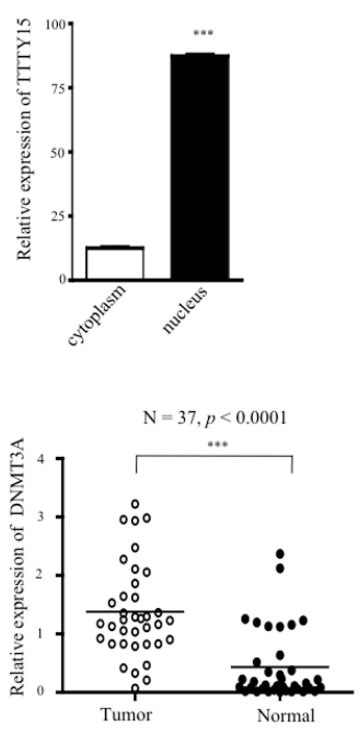

E.
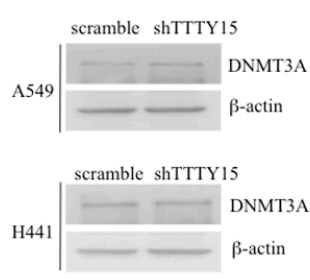

G.

1842 GAGAAAAGGAAGAGGCAAACCCTGAGCCCGGGAGGGAGAGCACTGAG CAGGCCTCGTCCCCAGTCCGAGCTGCGGGACCGGCGTCAGTGGACGCG GGCGGTGAACCAGCTCCTCACAGGGCAGGGCTGAGCGCGTAGGACTGA 8
GAGCGCAGGGCGCGAGCCGCAGGGCTCCGCTGCACGGCTCCGGGTGTG ACAAGAGCCCAGCAGAGGACCCCATG 15
B

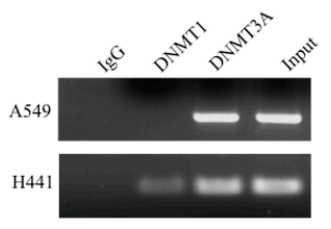

D.
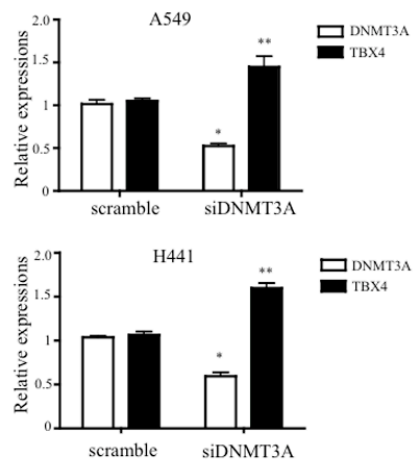

F.
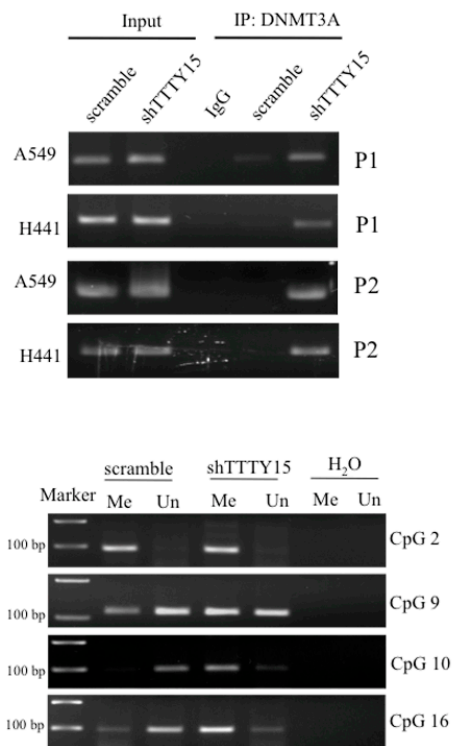

Figure 6. TTTY15 interacts with DNA methyltransferases (DNMT) 3A and decreases the binding of DNMT3A to the TBX4 promoter. (A) Cell fractionation assays were performed to determine the distribution of TTTY15 expression between the cell cytoplasm and nucleus. (B) TTTY15 RNA coimmunoprecipitated with DNMT1, DNMT3A, or IgG was quantified by qRT-PCR. PCR products were then loaded onto a 3\% agarose gel for confirmation. (C) DNMT3A turned out to be upregulated in human NSCLC samples compared to the adjacent normal tissue samples ( $n=37$ for each group). (D) The knockdown of DNMT3A increased the expression levels of TBX4 in A549 and H441 cells. (E) The knockdown of TTTY15 did not affect the protein expression levels of DNMT3A. (F) Chromatin immunoprecipitation (ChIP)-qPCR was performed to quantify the binding of DNMT3A to the TBX4 promoter (P1: -1734 to -1626 bp; P2: -740 to $-672 \mathrm{bp}$ ). (G) Methylation-specific PCR was performed to analyze the methylation status on the CPG islands of the TBX4 promoter in A549/scramble and A549/shTTTY15 (Me: methylated; Un: unmethylated; $\mathrm{H}_{2} \mathrm{O}$ was used as a negative control). Data are presented as the mean $\pm \mathrm{SD} ;{ }^{*} p<0.05,{ }^{* *} p<0.01,{ }^{* * *} p<0.001$. Statistical analysis was conducted by Student's $t$-test $(n=3)$. 


\section{Discussion}

Recent studies suggest that gender affects the risk, incidence, and progression of various human diseases, including cancers [20-24]. The most important genetic difference between men and women is the genes on the sex chromosomes. At present, there is a limited number of studies on $Y$ chromosome-linked lncRNAs in human diseases and cancers because the $\mathrm{Y}$ chromosome is often excluded from genomic analysis studies $[25,26]$. Our study provides the first evidence of the expression of a Y chromosome-linked lncRNA called TTTY15 in NSCLC. Some authors have reported that ubiquitin specific peptidase 9, Y-linked (USP9Y)-TTTY15 is expressed in both tumor and nonmalignant samples and can be used to predict the outcome of a prostate biopsy $[17,18,27]$. The TTTY15 gene can get fused to >zinc finger DHHC-type containing 2 (ZDHHC2-TTTY15), and this fusion is found in patients with acute myeloid leukemia [28]. In addition to forming fusion genes, TTTY15 (its product) can protect cardiomyocytes from hypoxia-induced cell injury by targeting miR-455-5p and thus regulating Jun dimerization protein 2 (JDP2) expression [29]. TTTY15 has also been reported to mainly localize in the cytoplasm and promote prostate cancer progression by functioning as an RNA sponge and binding to let-7 [30]. These studies show that TTTY15 plays important roles in cells. In our study, we found that TTTY15 was downregulated in lung cancer and was mainly located in the nucleus, upregulating TBX4 expression by targeting DNMT3A. These different findings suggest that TTTY15 may play a dual part in the cytoplasm and nucleus in various types of cancer. This study is the first to determine the clinical significance and biological function of TTTY15 in NSCLC, and the results indicate that TTTY15 is a tumor suppressor in NSCLC.

LncRNAs are long and can form complex secondary and tertiary structures to participate in gene regulation. A growing amount of research is suggesting that lncRNA genes can function as genes in certain situations and can regulate elements located on different chromosomes through processes involving the physical associations between different chromosomes ("trans-interactions") [31-34]. Chromosome interactions can promote the silencing and/or activation of genes within the three-dimensional structure of the nuclear matrix [35-37]. To elucidate the regulatory mechanism of action of TTTY15 in the NSCLC, we employed the 4C technique to identify the target regions of TTTY15 in the genome. The 4C data showed that TTTY15 targeted CCCD3 (chromosome 10) and LINC00674 (chromosome 17). After confirming the knockdown, we found that the knockdown of TTTY15 dramatically decreased the expression of T-box transcription factor 4 (TBX4). TBX4 is located in chromosomal region $17 \mathrm{q} 23$. Its protein product is reported to act as a transcription factor in hind limb growth and to regulate some processes during embryonic development [38,39]. TBX4- and TBX5-deficient mice show severely reduced lung branching during the second trimester, suggesting that TBX4 performs an important function in the regulation of proliferation, migration, and differentiation of mesenchymal cells, in addition to supporting and helping to produce peripheral epithelial cells [40]. In the present study, 4C was used in combination with next-generation sequencing and bioinformatic analysis to investigate the possible interactions of TTTY15 with other targets in the genome. This approach was effective at identifying the regulation targets of the lncRNA genes.

TBX4 is a mesenchymal transcription factor that drives the accumulation of myofibroblasts and the development of pulmonary fibrosis [41]. Low expression levels of TBX4 indicate a worse prognosis in patients with stage II pancreatic ductal adenocarcinoma (PDAC) [19]. Our results revealed that TBX4 is downregulated in NSCLC and the knockdown of TBX4 increases the NSCLC cell migration and invasion. MMPs are known to degrade proteins in the extracellular matrix and basement membrane, and to promote tumor metastasis [42]. Although the inhibition of the T-box transcription factor Brachyury is shown to downregulate MMP2 and MMP24 in cancer, to date [43], there are no reports on the direct participation of TBX4 in MMP gene expression. Therefore, we conducted experiments here to determine whether there is any correlation between MMP and TBX4. Our data showed that TBX4 silencing increases MMP9 expression in A549 and H441 cells. These results suggest that TBX4 is a tumor suppressor in NSCLC and inhibits the migration and invasiveness of NSCLC by decreasing the activity of MMP9. 
In addition to epigenetic control via chromosome modification, lncRNA can also affect gene regulation by its association with DNA methylation. Promoter methylation status is known to be involved in the initiation and progression of tumors. TBX4 is reported to be highly methylated in PDAC and human salivary adenoid cystic carcinoma $[19,44]$. In mammalian cells, DNMT3A and DNMT3B are known to establish a DNA methylation pattern de novo, while DNMT1 takes part in the maintenance of methylation status during DNA replication $[45,46]$. One study showed that lncRNA Dum interacts with three DNMTs to mediate and maintain local DNA methylation in the Dppa2 promoter [47]. In the present study, TTTY15 was found to regulate TBX4 expression via DNA methylation status. Our RNA immunoprecipitation and ChIP data revealed that TTTY15 can interact with DNMT3A and that the binding of DNMT3A to the promoter of TBX4 was blocked by TTTY15. Furthermore, the overexpression of a DNMT in a variety of tumors results in highly methylated and carcinogenically activated genes [48]. Moreover, either DNMT3A or DNMT3B is found in many clinical tumor samples, and the increased expression of DNMT3A was previously reported to participate in the progression of hepatocellular carcinoma [49,50]. Our results also showed that DNMT3A is overexpressed in NSCLC. These findings suggest that TTTY15 may interact with DNMT3A and decrease the ability of DNMT3A to bind to the TBX4 promoter.

\section{Materials and Methods}

\subsection{Patients and Sample Collection}

Thirty-seven paired NSCLC tissue samples and adjacent nontumor tissue samples from male patients (mean age 65.0, SD \pm 9.6) were obtained from the Biobank of China Medical University Hospital (CMUH) between 2006 and 2014. This study was conducted with the approval of the CMUH's Institutional Research Ethics Committee on 11 December 2014 (CMUH103-REC2-140), according to the Declaration of Helsinki's guidelines. None of the patients had received radiotherapy or chemotherapy prior to surgical resection. All tumor specimens were snap-frozen and stored in liquid nitrogen until analysis. The clinical and histopathological characteristics of each patient were also recorded. Informed written consent was obtained from all the patients.

\subsection{Cell Culture and Stable Transfection}

Cell lines A549, H441, H2170, and H520 were cultured in in Dulbecco's Modified Eagle Medium (DMEM) supplemented with 10\% fetal bovine serum (10\% FBS, Gibco, Gibco, Grand Island, NY, USA), $100 \mathrm{U} / \mathrm{mL}$ penicillin, and $100 \mu \mathrm{g} / \mathrm{mL}$ streptomycin in humidified air at $37^{\circ} \mathrm{C}$ with $5 \%$ of $\mathrm{CO}_{2}$. The medium was refreshed every day, and cell passaging was performed once every 3 days using $0.25 \%$ trypsin. The synthesis of shRNA (short hairpin RNA) downregulating TTTY15 was based on the pSUPER RNAi system expression vector (OligoEngine, Seattle, WA, USA). The oligo sequences designed for targeting TTTY15 were as follows: TTTY15-forward: 5'-GATCCCCTTTACAAAGAATTCCAGCTCTGTGGTTCAAGAGACCACAGAGCTGGAATTCTTT GTAAATTTTTA-3' and TTTY15-reverse: 5' -AGCTTAAAAATTTACAAAGAATTCCAGCTCTGTG GTCTCTTGAAGCCACAGAGCTGGAATTCTTTGTAAAGGG-3'. The annealing and ligation procedures were carried out according to the manufacturer's instructions. The A549 and H441 cells were transfected with the shTTTY15 plasmid and selected by means of G418. The efficiency of the TTTY15 knockdown was determined by qRT-PCR.

\subsection{SiRNA Transfection}

The siRNAs for targeting TBX4 and DNMT3A mRNA were constructed by MDBio Inc. (Taipei, Taiwan). The targeting sequences for TBX4 and DNMT3A were $5^{\prime}$-CCGAUGACCAUCGCUACAA TT- ${ }^{\prime}$ and $5^{\prime}$-CAGUGGUGUGUGUUGAGAATT- $3^{\prime}$, respectively. The scrambled ribooligonucleotide served as the negative control (a scrambled \%GC matched oligonucleotide). A549 and H441 cells were 
transfected with various doses of siRNA or a scramble ribooligonucleotide by means of RNAimax Lipofectamine (Invitrogen, Carlsbad, CA, USA), according to the manufacturer's instructions.

\subsection{Subcellular Fractionation, Total RNA Extraction, and qRT-PCR Analysis}

The nuclear and cytosolic fractions of the A549 and H441 cell lines were fractionated using the PARIS Kit (Life Technologies, Carlsbad, CA, USA), according to the manufacturer's instructions. Total RNA was extracted with the TRIzol reagent (Life Technologies, Scotland, UK), according to the manufacturer's instructions. The RNA was quantitated on a nanodrop spectrophotometer (Thermo Scientific, Waltham, MA, USA). RNA was reverse-transcribed into cDNA using the cDNA Reverse Transcriptase Kit (Thermo Fisher Scientific-Applied Biosystems, Waltham, MA, USA). qRT-PCR was performed via a TaqMan assay, where glyceraldehyde 3-phosphate dehydrogenase (GAPDH) served as the internal control for mRNA levels. The relative expression levels of the target genes were calculated as ratios normalized to GAPDH. The primers were synthesized by Genomics BioSci and Tech (Taipei, Taiwan). The following primer sequences were used (5'-3'): TTTY15-forward: tgagggagggatgtagctttt; TTTY15-reverse: gaagtcaagcaggcaactga; CCDC3-forward: tttctagccttttccagttttca; CCDC3-reverse: caaacaaggcettctgcac; LINC00674-forward: aagctgggctcaagagatcc; LINC00674-reverse: tggctgtggtggcttgta; KPNA2-forward: tgggccgtgaccaactatac; KPNA2-reverse: tgccacagtgaacaaggtaca; TBX4-forward: ccatcgctacaagttctgtgac; TBX4-reverse: gaatccgggtggacatacag; TIMP2-forward: gtgggtccaaggtcctcat; TIMP2-reverse: cgaagccccagacacatagt; DNMT3A-forward: cctgaagcctcaagagcagt; DNMT3A-reverse: tggtctcttctgttctttgc; MMP2-forward: ccccaaaacggacaaagag; MMP2-reverse: cttcagcacaaacaggttgc; $M M P 9$-forward: cgcagacatcgtcatccagt; $M M P 9$-reverse: cgcagacatcgtcatccagt. The average value of genes was measured using the $2^{-\Delta \Delta C t}$ method.

\subsection{A Cell Proliferation Assay}

This assay was conducted to determine whether the TTTY15 knockdown affected the viability of NSCLC cells. Briefly, TTTY15 stable knockdown A549 and H441 cells were seeded in 96-well plates (at 5000 cell/well). After 24, 48, and $72 \mathrm{~h}$, cell proliferation and viability were examined in an 3-(4,5-dimethylthiazol-2-yl)-2,5-diphenyltetrazolium bromide (MTT) assay. All the experiments were conducted in triplicate.

\subsection{Wound Healing Scratch and Transwell Invasion Assays}

A wound healing assay was performed to assess the migration ability of cells. A549 and H441 cells were seeded in a 24 -well plate as a monolayer at a density of $95-100 \%$. The cell monolayer was gently scratched across the center of the well with a $200 \mu \mathrm{L}$ plastic pipette tip. The rate of wound closure was observed by imaging by means of an inverted microscope (DMi1; Leica, Wetzlar, Germany). We then used the free software Tscratch to verify and calculate the migration movement of the entire wound area. A cell invasion assay was performed with transwell chambers $(8 \mu \mathrm{m}$ pore size), which were coated with $0.1 \%$ gelatin $\left(50 \mu \mathrm{L} /\right.$ well), in 24 -well plates. Approximately $10^{5}$ either scramble cells or shTTTY 15 cells were seeded into the upper chamber of the insert. After incubation for $24 \mathrm{~h}$ at $37^{\circ} \mathrm{C}$ with $5 \% \mathrm{CO}_{2}$, any cells that had invaded the membrane were stained with a $0.1 \%$ crystal violet solution. The cells were counted in three randomly selected visual fields under the inverted microscope (DMi1; Leica). For each experimental group, the assay was performed in triplicate.

\subsection{Flow Cytometry for Cell Cycle Analysis}

Transfected A549 and H441 cells were harvested $48 \mathrm{~h}$ after transfection. The cells were fixed in $70 \%$ ethanol at $-20^{\circ} \mathrm{C}$ overnight. The fixed cells were then washed once with phosphate-buffer-saline (PBS) and labeled with propidium iodide (Sigma-Aldrich, St. Louis, MO, USA) in the presence of RNase A (Sigma-Aldrich, St. Louis, MO, USA) and Triton X-100 for $30 \mathrm{~min}$ in the dark. The samples were run on a FACSCanto flow cytometer (BD Biosciences, San Diego, CA, USA) and the percentages of the cells within each phase of the cell cycle were analyzed in the ModFit LT software. 


\subsection{Circular Chromosome Conformation Capture}

As previously described, 4C was performed [51]. In brief, A549 cells were crosslinked with $1 \%$ formaldehyde for $10 \mathrm{~min}$ at room temperature to preserve the three-dimensional nuclear architecture. Sac I was used to digest the crosslinked chromatin (primary digestion). The digested chromatin was then ligated with the T4 DNA ligase. The digested and ligated chromatin was then de-crosslinked and subjected to the second restriction digestion using CviQ I to reduce the size of the fragments. Inverse PCRs were carried out with TTTY15-specific primers containing Illumina adapter sequences to amplify the genomic DNA fragments ligated to TTTY15 (first PCR: forward 5'-tggtgcgatcttgatttactgc-3' , reverse 5'-tgcctttgtctgtatgt gca-3'; nested PCR: forward $5^{\prime}$-tcattcttgttgcccagtctg- $3^{\prime}$, reverse $5^{\prime}$-ttcacctttgttggctccc- $\left.3^{\prime}\right)$.

\subsection{Next-Generation Sequencing and Bioinformatic Analysis of TTTY15 4C-Sequencing Data}

The PCR products were purified with the Qiagen Mini-Elute kit (Qiagen, Hilden, Germany). The amplicon was then prepared for sequencing using the TruSeq DNA library preparation kit (Illumina, San Diego, California). After that, $100 \mathrm{ng}$ purified amplicon pools were repaired to generate blunt-ended ligations, according to the TruSeq DNA Sample Preparation protocol. 5'-Phosphorylated DNA and an A-tailing reaction compatible with the adapter ligation strategy were performed. The ligation product was then purified using sample purification beads. In order to enrich the library, an enhanced PCR mix was used for PCR amplification. The size distribution of the library was verified using the High-Sensitivity DNA Kit (Agilent, Technologies, Waldbronn, Germany), and the concentration of the library was quantified with the GeneRead Library Quant Kit (Qiagen, Hilden, Germany). The library was diluted and sequenced with 500 paired-end cycles on the Illumina MiSeq platform by following the standard protocol. For bioinformatic analysis, any known fragments were removed from the sequencing reads as follows. First, the forward and reverse sequencing reads were merged into one sequence, such that the length of the overlapping region was over 20 nucleotides. Next, the bioinformatic software of sequence BLAST was used to identify the location of the known fragments and primers [52]. The known fragments were located in the regions between the primers and the cutting site and the alignment similarity for BLAST was set to 95\%. Finally, these primers and known fragments of sequencing reads were removed from the sequences. The remaining region of the sequences was labeled as "unknown fragments". The Bowtie2 software is an efficient tool for the identification of potential TTTY15 interaction regions, therefore by aligning sequencing reads against reference sequences [53], we aligned unknown fragments against human genome sequences (Grch38.p2 was employed in the present study) in this software. Subsequently, fourSig was used to identify the potential TTTY15-interacting genome regions [54]. After scanning for the interacting regions, fourSig provided two categories of regions that were defined as potential TTTY15 interaction regions. The regions and their associated genes were listed and annotated according to the known human genomic location. These two interaction regions were used as the targeting candidates for TTTY15.

\subsection{The Western Blot Assay}

Whole-cell extracts were prepared from A549 and $\mathrm{H} 441$ cells by adding radioimmunoprecipitation assay (RIPA) lysis buffer (150 mM NaCl, 0.1\% SDS, 0.5\% sodium deoxycholate, 1\% NP-40) (Sigma-Aldrich, St. Louis, MO, USA) with complete protease inhibitor cocktails (Sigma). Equal quantities of total protein samples were separated on 10\% SDS-PAGE gels and transferred onto poly vinylidene fluoride (PVDF) membranes. The blots were incubated with primary antibody against DNMT3A (ab13888, Abcam, Cambridge, MA, USA) overnight at $4{ }^{\circ} \mathrm{C}$. After second antibody incubation, the electrochemiluminescence (ECL) kit (EMD Millipore, St. Charles, MO, USA) was utilized to visualize the protein signals. $\beta$-Actin (Proteintec, Rosemont, IL, USA) served as the internal control. 


\subsection{RNA Immunoprecipitation}

This procedure was carried out using ChIP-IT Kit (Active Motif, Carlsbad, CA, USA), according to the manufacturer's instructions. Briefly, endogenous DNMT1 and DNMT3A complexes from the whole-cell extract were pulled down using anti-DNMT1 (ab13537) or anti-DNMT3A (ab13888, Abcam, Cambridge, MA, USA) antibody-coated beads. The beads were then washed with wash buffer and eluted with elution buffer. The eluted samples were incubated with $0.5 \mathrm{mg} / \mathrm{mL}$ protease $\mathrm{K}$ to remove proteins. The isolate from the immunoprecipitation product was further validated by qRT-PCR.

\subsection{Chromatin Immunoprecipitation}

The DNA ChIP assay was performed using the ChIP-IT Kit (Active Motif, Carlsbad, CA, USA) according to the manufacturer's instructions. Briefly, anti-DNMT1 and anti-DNMT3A antibody-coated beads were used to pull down the DNMT1 and DNMT3A complexes from A549 and H441 cells. The beads were washed three times with washing buffer. The beads were then eluted and subjected to reverse crosslinking. The isolate from the IP product was then validated by qRT-PCR. The following primers were designed to amplify the TBX4 promoter region: TBX4 promoter ( -749 to $-672 \mathrm{bp})$-forward: $5^{\prime}$-cagagctggatcagtcacc- $3^{\prime}$, reverse: $5^{\prime}$-tctggcacagacatcctcac- $3^{\prime}$ and TBX4 promoter $(-1734$ to -1626 bp)-forward: 5'-TGAACCAGCTCCTCACAGG-3', reverse: 5'-CTCTGCTGGGCTCTTG TCAC-3' .

\subsection{Methylation-Specific PCR}

The methylation status of the promoter region on the TBX4 gene (from -1842 to $-1626 \mathrm{bp}$ ) was analyzed by methylation-specific PCR. Briefly, the genomic DNA of A549 cells was extracted using the QIAamp DNA Mini Kit (Qiagen, Germany) and was then modified with the Zymo EZ DNA Methylation Kit (Zymo Research, Tustin, CA, USA), according to the manufacturer's instructions. The modified DNA was amplified using a $20 \mu \mathrm{L}$ mixture including HotStarTag buffer $\left(2.0 \mathrm{mM} \mathrm{Mg}^{2+}\right.$, $0.2 \mathrm{mM}$ deoxy-ribonucleoside triphosphate (dNTP), 1 U HotStarTag polymerase (Qiagen, Germany)), $0.2 \mu \mathrm{M}$ of each primer and $1 \mu \mathrm{L}$ of template DNA. The amplification program for methylation-specific PCR was as follows: an initial incubation for $10 \mathrm{~min}$ at $95^{\circ} \mathrm{C}$, followed by 35 cycles of $95{ }^{\circ} \mathrm{C}$ for $30 \mathrm{~s}, 50^{\circ} \mathrm{C}$ for $1 \mathrm{~min}, 72{ }^{\circ} \mathrm{C}$ for $1 \mathrm{~s}$, and a final $5 \mathrm{~min}$ incubation at $72{ }^{\circ} \mathrm{C}$. The primer information for methylation-specific PCR was shown in Table S3.

\subsection{Statistical Analysis}

All experimental data from the three independent experiments were analyzed in GraphPad Prism version 5 (GraphPad Software Inc., La Jolla, CA, USA). The results were expressed as the mean \pm SD (standard deviation). The associations between the relative TTTY15 RNA expression levels and the clinical parameters (age, tumor size, lymph node metastasis, and TNM stage) were analyzed by Fisher's exact test. A Student's $t$-test was conducted to analyze the differences, where $p<0.05$ was assumed to indicate a statistically significant difference.

\section{Conclusions}

The Y chromosome contains not only genes involved in sex determination but also genes that participate in the prevention of tumors. This study is the first to provide evidence for the potential involvement of the Y chromosome-linked TTTY15 in NSCLC. We found that TTTY15 is downregulated in NSCLC and was associated with lymph node metastasis and the TNM stage of patients with NSCLC. Our study determined a novel target gene of TTTY15 and revealed the interactions among TTTY15, DNMT3A, TBX4, and MMP9 in NSCLC cells. The application of the 4C technique allowed for a genome-wide search for lncRNA target genes and may facilitate further studies on the function of lncRNAs in cancer. Our analysis of the $\mathrm{Y}$ chromosome could be conducted to predict the risk of cancer in men. 
Supplementary Materials: Supplementary materials can be found at http://www.mdpi.com/1422-0067/20/14/ 3473/s1.

Author Contributions: Conceptualization, I-L.L. and J.-G.C.; data curation, I-L.L., Y.-T.L. and J.-C.Y.; formal analysis, I-L.L., W.-L.C. and Y.-T.L.; funding acquisition, C.-A.Y. and J.-G.C.; investigation, I-L.L., Y.-S.C., C.-A.Y., and S.-Y.H.; methodology, I-L.L., Y.-S.C., W.-L.C., Y.-T.L., and J.-C.Y.; project administration, I.-L.L., Y.-S.C., and Y.-T.L.; resources, C.-A.Y. and J.-G.C.; supervision, J.-G.C.; validation, W.-L.C.,Y.-T.L. and J.-C.Y.; visualization, I-L.L.; writing—original draft, S.-Y.H.; writing—review and editing, J.-G.C.

Funding: This work was supported by the grant to the Ministry of Science and Technology (MOST), Taiwan, grant (103-2320-B-039-050-MY3, 106-2320-B-039-043-MY3, 106-2221-E-468-018, and 107-2221-E-468-01), China Medical University Hospital (CMUH 102-REC1-037 and DMR-104-101), and Asia University (ASIA-107-CMUH-117, and ASIA-107-AUH-08).

Acknowledgments: We thank David Alder for the drawing of gene locus for chromosome 17 in Figure 4C. http://www.pathology.washington.edu/research/cytopages/idiograms/human/. We thank Yu-Hsuan Juan for graphical assistance.

Conflicts of Interest: The authors declare no conflicts of interest.

\section{References}

1. Lundin, A.; Driscoll, B. Lung cancer stem cells: Progress and prospects. Cancer letters 2013, 338, 89-93. [CrossRef] [PubMed]

2. Xue, W.; Dahlman, J.E.; Tammela, T.; Khan, O.F.; Sood, S.; Dave, A.; Cai, W.; Chirino, L.M.; Yang, G.R.; Bronson, R.; et al. Small RNA combination therapy for lung cancer. Proc. Natl. Acad. Sci. USA 2014, 111, E3553-E3561. [CrossRef] [PubMed]

3. Bareschino, M.A.; Schettino, C.; Rossi, A.; Maione, P.; Sacco, P.C.; Zeppa, R.; Gridelli, C. Treatment of advanced non small cell lung cancer. J. Thorac. Dis. 2011, 3, 122-133. [PubMed]

4. Navab, R.; Strumpf, D.; Bandarchi, B.; Zhu, C.Q.; Pintilie, M.; Ramnarine, V.R.; Ibrahimov, E.; Radulovich, N.; Leung, L.; Barczyk, M.; et al. Prognostic gene-expression signature of carcinoma-associated fibroblasts in non-small cell lung cancer. Proc. Natl. Acad. Sci. USA 2011, 108, 7160-7165. [CrossRef]

5. Jemal, A.; Bray, F.; Center, M.M.; Ferlay, J.; Ward, E.; Forman, D. Global cancer statistics. Cancer J. Clin. 2011, 61, 69-90. [CrossRef]

6. Pao, W.; Girard, N. New driver mutations in non-small-cell lung cancer. Lancet 2011, 12, 175-180. [CrossRef]

7. Sandberg, A.A. Chromosomal abnormalities and related events in prostate cancer. Hum. Pathol. 1992, 23, 368-380. [CrossRef]

8. $\quad$ Lundgren, R.; Mandahl, N.; Heim, S.; Limon, J.; Henrikson, H.; Mitelman, F. Cytogenetic analysis of 57 primary prostatic adenocarcinomas. Genes Chromosomes Cancer 1992, 4, 16-24. [CrossRef]

9. Sauter, G.; Moch, H.; Wagner, U.; Novotna, H.; Gasser, T.C.; Mattarelli, G.; Mihatsch, M.J.; Waldman, F.M. Y chromosome loss detected by FISH in bladder cancer. Cancer Genet. Cytogenet. 1995, 82, 163-169. [CrossRef]

10. Wada, M.; Yokota, J.; Mizoguchi, H.; Terada, M.; Sugimura, T. Y chromosome abnormality in human stomach and lung cancer. Jpn. J. Cancer Res. 1987, 78, 780-783.

11. Center, R.; Lukeis, R.; Vrazas, V.; Garson, O.M. Y chromosome loss and rearrangement in non-small-cell lung cancer. Int. J. Cancer 1993, 55, 390-393. [CrossRef] [PubMed]

12. Hunter, S.; Gramlich, T.; Abbott, K.; Varma, V. Y chromosome loss in esophageal carcinoma: An in situ hybridization study. Genes Chromosomes Cancer 1993, 8, 172-177. [CrossRef] [PubMed]

13. Dorak, M.T.; Karpuzoglu, E. Gender differences in cancer susceptibility: An inadequately addressed issue. Front. Genet. 2012, 3, 268. [CrossRef] [PubMed]

14. Skaletsky, H.; Kuroda-Kawaguchi, T.; Minx, P.J.; Cordum, H.S.; Hillier, L.; Brown, L.G.; Repping, S.; Pyntikova, T.; Ali, J.; Bieri, T.; et al. The male-specific region of the human Y chromosome is a mosaic of discrete sequence classes. Nature 2003, 423, 825-837. [CrossRef] [PubMed]

15. Graves, J.A.; Koina, E.; Sankovic, N. How the gene content of human sex chromosomes evolved. Curr. Opin. Genet. Dev. 2006, 16, 219-224. [CrossRef] [PubMed]

16. Molina, E.; Chew, G.S.; Myers, S.A.; Clarence, E.M.; Eales, J.M.; Tomaszewski, M.; Charchar, F.J. A Novel Y-Specific Long Non-Coding RNA Associated with Cellular Lipid Accumulation in HepG2 cells and Atherosclerosis-related Genes. Sci. Rep. 2017, 7, 16710. [CrossRef] [PubMed] 
17. Zhu, Y.; Ren, S.; Jing, T.; Cai, X.; Liu, Y.; Wang, F.; Zhang, W.; Shi, X.; Chen, R.; Shen, J.; et al. Clinical utility of a novel urine-based gene fusion TTTY15-USP9Y in predicting prostate biopsy outcome. Urol. Oncol. 2015, 33, 384. [CrossRef] [PubMed]

18. Ren, S.; Peng, Z.; Mao, J.H.; Yu, Y.; Yin, C.; Gao, X.; Cui, Z.; Zhang, J.; Yi, K.; Xu, W.; et al. RNA-seq analysis of prostate cancer in the Chinese population identifies recurrent gene fusions, cancer-associated long noncoding RNAs and aberrant alternative splicings. Cell Res. 2012, 22, 806-821. [CrossRef] [PubMed]

19. Zong, M.; Meng, M.; Li, L. Low expression of TBX4 predicts poor prognosis in patients with stage II pancreatic ductal adenocarcinoma. Int. J. Mol. Sci. 2011, 12, 4953-4963. [CrossRef]

20. Postma, D.S. Gender differences in asthma development and progression. Gender Med. 2007, 4, S133-S146. [CrossRef]

21. Ngo, S.T.; Steyn, F.J.; McCombe, P.A. Gender differences in autoimmune disease. Front. Neuroendocrinol. 2014, 35, 347-369. [CrossRef] [PubMed]

22. Luczak, E.D.; Leinwand, L.A. Sex-based cardiac physiology. Annu. Rev. Physiol. 2009, 71, 1-18. [CrossRef] [PubMed]

23. Nakamura, H.; Ando, K.; Shinmyo, T.; Morita, K.; Mochizuki, A.; Kurimoto, N.; Tatsunami, S. Female gender is an independent prognostic factor in non-small-cell lung cancer: A meta-analysis. Ann. Thor. Cardiovasc. Surg. 2011, 17, 469-480. [CrossRef] [PubMed]

24. Fajkovic, H.; Halpern, J.A.; Cha, E.K.; Bahadori, A.; Chromecki, T.F.; Karakiewicz, P.I.; Breinl, E.; Merseburger, A.S.; Shariat, S.F. Impact of gender on bladder cancer incidence, staging, and prognosis. World J. Urol. 2011, 29, 457-463. [CrossRef] [PubMed]

25. Guttman, M.; Amit, I.; Garber, M.; French, C.; Lin, M.F.; Feldser, D.; Huarte, M.; Zuk, O.; Carey, B.W.; Cassady, J.P.; et al. Chromatin signature reveals over a thousand highly conserved large non-coding RNAs in mammals. Nature 2009, 458, 223-227. [CrossRef] [PubMed]

26. Guttman, M.; Donaghey, J.; Carey, B.W.; Garber, M.; Grenier, J.K.; Munson, G.; Young, G.; Lucas, A.B.; Ach, R.; Bruhn, L.; et al. lincRNAs act in the circuitry controlling pluripotency and differentiation. Nature 2011, 477, 295-300. [CrossRef] [PubMed]

27. Ren, G.; Zhang, Y.; Mao, X.; Liu, X.; Mercer, E.; Marzec, J.; Ding, D.; Jiao, Y.; Qiu, Q.; Sun, Y.; et al. Transcription-mediated chimeric RNAs in prostate cancer: Time to revisit old hypothesis? Omics J. Integr. Biol. 2014, 18, 615-624. [CrossRef] [PubMed]

28. Wang, L.; Sun, Y.; Sun, Y.; Meng, L.; Xu, X. First case of AML with rare chromosome translocations: A case report of twins. BMC Cancer 2018, 18, 458. [CrossRef] [PubMed]

29. Huang, S.; Tao, W.; Guo, Z.; Cao, J.; Huang, X. Suppression of long noncoding RNA TTTY15 attenuates hypoxia-induced cardiomyocytes injury by targeting miR-455-5p. Gene 2019, 701, 1-8. [CrossRef]

30. Xiao, G.; Yao, J.; Kong, D.; Ye, C.; Chen, R.; Li, L.; Zeng, T.; Wang, L.; Zhang, W.; Shi, X.; et al. The Long Noncoding RNA TTTY15, Which Is Located on the Y Chromosome, Promotes Prostate Cancer Progression by Sponging let-7. Eur. Urol. 2018. [CrossRef]

31. Spilianakis, C.G.; Lalioti, M.D.; Town, T.; Lee, G.R.; Flavell, R.A. Interchromosomal associations between alternatively expressed loci. Nature 2005, 435, 637-645. [CrossRef] [PubMed]

32. Lomvardas, S.; Barnea, G.; Pisapia, D.J.; Mendelsohn, M.; Kirkland, J.; Axel, R. Interchromosomal interactions and olfactory receptor choice. Cell 2006, 126, 403-413. [CrossRef] [PubMed]

33. Xu, N.; Tsai, C.L.; Lee, J.T. Transient homologous chromosome pairing marks the onset of $X$ inactivation. Science 2006, 311, 1149-1152. [CrossRef] [PubMed]

34. Bacher, C.P.; Guggiari, M.; Brors, B.; Augui, S.; Clerc, P.; Avner, P.; Eils, R.; Heard, E. Transient colocalization of X-inactivation centres accompanies the initiation of X inactivation. Nat. Cell Biol. 2006, 8, 293-299. [CrossRef]

35. Galande, S.; Purbey, P.K.; Notani, D.; Kumar, P.P. The third dimension of gene regulation: Organization of dynamic chromatin loopscape by SATB1. Curr. Opin. Genet. Dev. 2007, 17, 408-414. [CrossRef] [PubMed]

36. Gondor, A.; Ohlsson, R. Chromosome crosstalk in three dimensions. Nature 2009, 461, 212-217. [CrossRef] [PubMed]

37. Cavalli, G.; Misteli, T. Functional implications of genome topology. Nat. Struct. Mol. Biol. 2013, 20, $290-299$. [CrossRef]

38. Agulnik, S.I.; Garvey, N.; Hancock, S.; Ruvinsky, I.; Chapman, D.L.; Agulnik, I.; Bollag, R.; Papaioannou, V.; Silver, L.M. Evolution of mouse T-box genes by tandem duplication and cluster dispersion. Genetics 1996, 144, 249-254. 
39. Naiche, L.A.; Papaioannou, V.E. Loss of Tbx4 blocks hindlimb development and affects vascularization and fusion of the allantois. Development 2003, 130, 2681-2693. [CrossRef]

40. Arora, R.; Metzger, R.J.; Papaioannou, V.E. Multiple roles and interactions of Tbx4 and Tbx5 in development of the respiratory system. PLoS Genet. 2012, 8, e1002866. [CrossRef]

41. Xie, T.; Liang, J.; Liu, N.; Huan, C.; Zhang, Y.; Liu, W.; Kumar, M.; Xiao, R.; D'Armiento, J.; Metzger, D.; et al. Transcription factor TBX4 regulates myofibroblast accumulation and lung fibrosis. J. Clin. Investig. 2016, 126, 3626. [CrossRef] [PubMed]

42. Egeblad, M.; Werb, Z. New functions for the matrix metalloproteinases in cancer progression. Nat. Rev. Cancer 2002, 2, 161-174. [CrossRef] [PubMed]

43. Fernando, R.I.; Litzinger, M.; Trono, P.; Hamilton, D.H.; Schlom, J.; Palena, C. The T-box transcription factor Brachyury promotes epithelial-mesenchymal transition in human tumor cells. J. Clin. Investig. 2010, 120, 533-544. [CrossRef] [PubMed]

44. Bell, A.; Bell, D.; Weber, R.S.; El-Naggar, A.K. CpG island methylation profiling in human salivary gland adenoid cystic carcinoma. Cancer 2011, 117, 2898-2909. [CrossRef] [PubMed]

45. Dekker, J. A closer look at long-range chromosomal interactions. Trends Biochem. Sci. 2003, 28, $277-280$. [CrossRef]

46. Stamatoyannopoulos, G. Control of globin gene expression during development and erythroid differentiation. Exp. Hematol. 2005, 33, 259-271. [CrossRef]

47. Wang, L.; Zhao, Y.; Bao, X.; Zhu, X.; Kwok, Y.K.; Sun, K.; Chen, X.; Huang, Y.; Jauch, R.; Esteban, M.A.; et al. LncRNA Dum interacts with Dnmts to regulate Dppa2 expression during myogenic differentiation and muscle regeneration. Cell Res. 2015, 25, 335-350. [CrossRef]

48. Esteller, M. Epigenetics in cancer. N. Engl. J. Med. 2008, 358, 1148-1159. [CrossRef]

49. Zhao, Z.; Wu, Q.; Cheng, J.; Qiu, X.; Zhang, J.; Fan, H. Depletion of DNMT3A suppressed cell proliferation and restored PTEN in hepatocellular carcinoma cell. J. Biomed. Biotechnol. 2010, 2010, 737535. [CrossRef]

50. Deng, T.; Kuang, Y.; Wang, L.; Li, J.; Wang, Z.; Fei, J. An essential role for DNA methyltransferase 3a in melanoma tumorigenesis. Biochem. Biophys. Res. Commun. 2009, 387, 611-616. [CrossRef]

51. Stadhouders, R.; Kolovos, P.; Brouwer, R.; Zuin, J.; van den Heuvel, A.; Kockx, C.; Palstra, R.J.; Wendt, K.S.; Grosveld, F.; van Ijcken, W.; et al. Multiplexed chromosome conformation capture sequencing for rapid genome-scale high-resolution detection of long-range chromatin interactions. Nat. Protocols 2013, 8, 509-524. [CrossRef] [PubMed]

52. Altschul, S.F.; Gish, W.; Miller, W.; Myers, E.W.; Lipman, D.J. Basic local alignment search tool. J. Mol. Biol. 1990, 215, 403-410. [CrossRef]

53. Langmead, B.; Trapnell, C.; Pop, M.; Salzberg, S.L. Ultrafast and memory-efficient alignment of short DNA sequences to the human genome. Genome Biol. 2009, 10, R25. [CrossRef] [PubMed]

54. Williams, R.L., Jr.; Starmer, J.; Mugford, J.W.; Calabrese, J.M.; Mieczkowski, P.; Yee, D.; Magnuson, T. fourSig: A method for determining chromosomal interactions in 4C-Seq data. Nucleic Acids Res. 2014, 42, e68. [CrossRef] [PubMed]

(C) 2019 by the authors. Licensee MDPI, Basel, Switzerland. This article is an open access article distributed under the terms and conditions of the Creative Commons Attribution (CC BY) license (http://creativecommons.org/licenses/by/4.0/). 